\section{Reality of bubble nuclei}

from our Nuclear Theory Correspondent

MANY studies of nuclei by electron and nucleon scattering have shown that some are spherical whereas others are more or less strongly deformed into spheroidal or ellipsoidal shapes. Studies of their excited states show that the deformed nuclei have characteristic rotational spectra but that the spherical nuclei can often be excited into a series of vibrational modes.

Wheeler pointed out long ago that these are not the only conceivable nuclear shapes. One can imagine not only wobbly drops of various shapes but more exotic configurations like bubbles and toroids. Is it possible, for example, to form a bubble of nuclear matter and if so would it be stable?

It is plainly possible to imagine a nucleus from which all the nucleons in the lowest ls state have been suddenly removed by a direct interaction, leaving the other nucleons undisturbed. This would be a highly excited nucleus but it might live long enough to qualify as a bubble nucleus. It is also conceivable that bubble nuclei are sometimes formed in the turbulent nuclear motions resulting from very energetic collisions of heavy ions. These bubble nuclei are likely to be highly excited but it is also possible that bubble configurations are found at low excitations or even in the ground state.

Whereas heavy nuclei can usefully be thought of for some purposes as a drop of fluid of almost uniform density, light nuclei have density distributions that can be interpreted as $\alpha$-like clusters in regular array. Thus ${ }^{12} \mathrm{C}$ has a density distribution similar to that of three $\alpha \mathrm{s}$ at the vertices of an equilateral triangle and ${ }^{16} \mathrm{O}$ as four at the vertices of a tetrahedron. Such nuclei may have a low central density and may be classed as bubble nuclei.

Calculations of the stability of bubble nuclei were made by Siemens and Bethe (Phys. Rev. Lett., 18, 704; 1967) using the semi-empirical mass formula and they found that some large spherical bubble nuclei may be stable against symmetry-preserving breathing expansions and contractions, but they did not investigate their stability against other distortions.

The stability of bubble-shaped nuclei against symmetry-preserving breathing deformations has also been investigated by Krishnan and $\mathrm{Pu}$ (Phys. Lett., 47B, 225 ; 1973) using the energy density formalism. They find that bubble nuclei are stable only for very large mass numbers $(A \simeq 700)$, far beyond the region known at present. Lighter nuclei do not have stable bubbles.

A series of calculations using the liquid drop model shows that bubble nuclei are unstable (Wong, Ann. Phys., 77, 279; 1973) and this confirms other calculations that do not take the shell structure of nuclei into account. If, however, the shell structure is included, some bubble nuclei with a low density of singleparticle states near the Fermi surface are found to be stable. These calculations are made by assuming that the nuclear potential follows the density distribution; the energies of the single-particle states can then be found by standard procedures. The reduced potential near the centre of a bubble nucleus raises the energies of those single-particle states whose wave functions overlap with this region, and these are states of low angular momentum. Thus in a bubble configuration the energies of states of low angular momentum are raised relative to those of states with high angular momentum and this gives a new series of magic nucleon numbers for pronounced bubble configurations.

In this way Wong (Phys. Lett. 41B, $451 ; 1972$ ) found the new magic numbers $18,34,50,70,80,104,120 \ldots$ at various deformations. Use of the Strutinsky method to evaluate the shell corrections then indicates ${ }^{36} \mathrm{Ar},{ }^{84} \mathrm{Se},{ }^{138} \mathrm{Ce}$ and ${ }^{200} \mathrm{Hg}$ as possible bubble nuclei in their ground states.

More detailed calculations may be made with the Hartree-Fock theory using a realistic effective nucleon interaction and some results of Curry and Sprung (Nucl. Phys., A216, 125; 1973) for ${ }^{36} \mathrm{Ar}$ are shown in Fig. 1. Also shown is the corresponding distribution for ${ }^{40} \mathrm{Ca}$ less that of an $\alpha$ particle. Allowing for

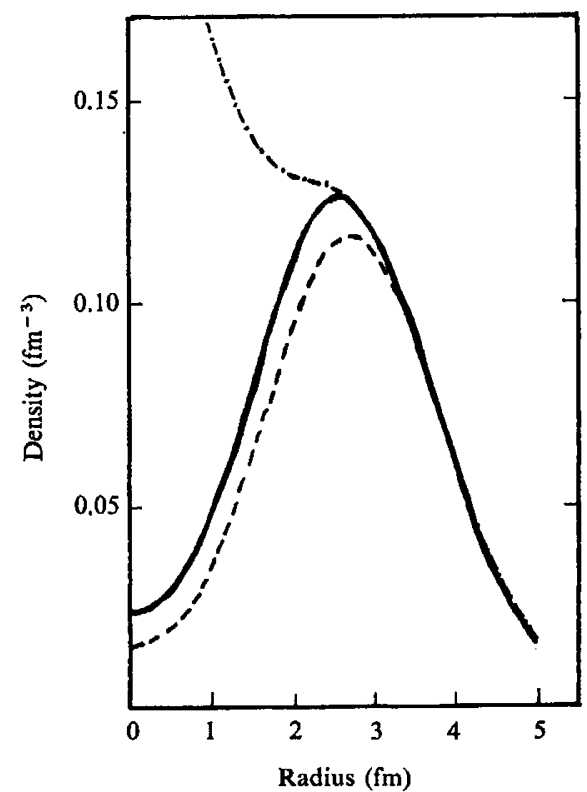

Fig. 1 Density distribution of ${ }^{36} \mathrm{Ar}$ (- ) compared with that of ${ }^{40} \mathrm{Ca}$ $(-\bullet-\bullet)$ and the density difference of ${ }^{40} \mathrm{Cal}$ and an $\alpha$ particle $(---)$ obtained by Hartree-Fock calculations. The central bubble is well marked, and is identified as an $\alpha$ hole (Curry and Sprung).

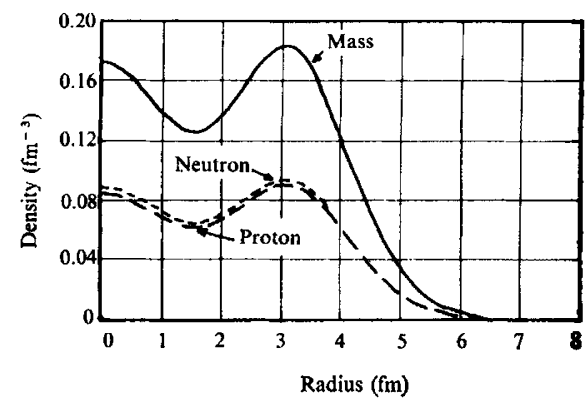

Fia. 2 Density distribution of $68 \mathrm{Se}$ showing the region of minimum density centred on a spherical shell of radius about $1.5 \mathrm{fm}$ (Davies, Krieger and Wong).

a slight inward relaxation due to selfconsistency effects the two distributions correspond very well, suggesting that ${ }^{36} \mathrm{Ar}$ has a bubble configuration corresponding to ${ }^{40} \mathrm{Ca}$ with an $\alpha$ particle removed from its centre. These nucleons in the $\alpha$ particle are not, however, in $s$ states; removal of s-state nucleons would give ${ }^{36} \mathrm{Ar}$ in a highly excited state and a density distribution quite different from that shown.

Further calculations for a series of possible bubble nuclei have been made by Davies, Krieger and Wong ( $N u c l$. Phys., A216, 250; 1973) and they confirm the bubble nature of a low energy configuration of ${ }^{36} \mathrm{Ar}$ and in addition find marked bubble effects in ${ }^{200} \mathrm{Hg}$ and ${ }^{116} \mathrm{Ce}$ and ${ }^{138} \mathrm{Ce}$. A different type of bubble is found in ${ }^{68} \mathrm{Se}$; the region of lower density is centred on a spherical shell of radius about $1.5 \mathrm{fm}$ instead of the origin (see Fig. 2).

Detailed examination of the results of these calculations shows that in these nuclei the bubble configurations are preferred because they reduce the density of single-particle states in the region of the Fermi surface. The expected raising of states of low angular momentum relative to those of high angular momentum is indeed found in some bubble nuclei. With the same model, the strength of the spin-orbit potential of the Thomas form (proportional to the radial derivative of the density distribution) depends on the relative amplitudes for finding the nucleon at the surface or near the centre of the nucleus. This implies that for bubble nuclei the spinorbit doublets may be inverted and the likelihood of this is greater for smaller values of orbital angular momentum. This effect is also observed in some nuclei.

These investigations show that the usual picture of nuclei having an almost uniform central density is sometimes far from the truth. There are several nuclei that show pronounced minima or bubbles in their centres and others have even more complicated departures from uniform density. 